Check for updates

Cite this: RSC Adv., 2017, 7, 27058

\title{
Oxide-derived nanostructured metallic-glass electrodes for efficient electrochemical hydrogen generation $\uparrow$
}

\author{
Shenghan Gao, (D) Jili Jia, Shuangqin Chen, (D) Hengwei Luan, (D) Yang Shao (D)* \\ and Kefu Yao
}

Nanostructured materials with large surface areas are continuing to be at the forefront of catalytic applications. But the nanostructure synthesis methods are often lengthy, costly and difficult. Here we report the first successful fabrication of metallic-glass (MG) hierarchical nanostructures by combining thermoplastic forming and electrochemical reduction process. By the simple synthesis technique, the oxide-derived MG nanorod arrays (OD-MG NRAs) electrode with higher specific surface area exhibits an enhanced catalytic activity towards hydrogen evolution reaction than that of untreated flat MG. The ODMG NRAs electrode demonstrates efficient electrochemical hydrogen generation in an acidic electrolyte (10 mA cm ${ }^{-2}$ at overpotential of $63 \mathrm{mV}$; Tafel slope of $42.6 \mathrm{mV} \mathrm{dec}^{-1}$ ) and possesses impressive selfstabilizing catalytic activity over a long-term operation. These features promise an attractive catalyst for large-scale hydrogen production.

Received 11th March 2017 Accepted 15th May 2017

DOI: $10.1039 / c 7 r a 02954 c$

rsc.li/rsc-advances with morphologies necessary for maintaining high dispersion and noble metal utilization.

Metallic glasses (MGs) or amorphous alloys are well-known for their outstanding mechanical properties and recently have been suggested to be potential candidates for electrocatalytic applications. ${ }^{\mathbf{1 5 - 2 0}}$ They are multicomponent metallic alloys with a wide range of chemical compositions, including catalytic noble metals such as platinum or palladium..$^{15,16,21-24}$ Due to their amorphous nature and unique composition, metallicglass can be thermoplastically formed into complex structures of nano- to micro-sized features. ${ }^{18,20,25-28}$ Recently, through thermoplastic forming, Schroers, Taylor and co-workers demonstrated nanowire MGs as effective electrocatalysts for methanol/ethanol oxidation or oxygen reduction reaction. ${ }^{\mathbf{1 8 , 2 0 , 2 9}}$ Zhao et al. discovered that MG nanoparticles exhibited remarkable catalytic properties in methanol electro-oxidation. ${ }^{\mathbf{1 6}}$

This study is the first exploration of the catalytic activity of the electrochemical production of hydrogen on oxide-derived nanostructured MG catalyst. Herein we report the synthesis of a novel surface-modified $\mathrm{Pd}_{40.5} \mathrm{Ni}_{40.5} \mathrm{Si}_{4.5} \mathrm{P}_{14.5} \mathrm{MG}$ nanorod arrays electrocatalyst for HER. By combining thermoplastic forming together with electroreduction of oxide films on the surface, hierarchical metallic glass nanostructures with large specific surface area and high catalytic activity are synthesized. Oxide-derived MG nanorod arrays (denoted as OD-MG NRAs) electrode achieved a geometrical catalytic current density of 10 $\mathrm{mA} \mathrm{cm}{ }^{-2}$ at overpotential of $63 \mathrm{mV}$, with unique self-enhancing long-term operational stability. Moreover, the overpotential of this OD-MG NRAs electrode was only $99 \mathrm{mV}$ driving a current
School of Materials Science and Engineering, Tsinghua University, Beijing 100084, People's Republic of China. E-mail: shaoyang@tsinghua.edu.cn

$\dagger$ Electronic supplementary information (ESI) available. See DOI: $10.1039 / \mathrm{c} 7 \mathrm{ra02954c}$ 
density of $j=50 \mathrm{~mA} \mathrm{~cm}{ }^{-2}$, while the potential of the untreated flat MG plate reached $227 \mathrm{mV}$ at the same condition.

\section{Experimental}

\section{Synthesis of OD-MG NRAs electrode}

Metallic glass with nominal composition of $\mathrm{Pd}_{40.5} \mathrm{Ni}_{40.5} \mathrm{Si}_{4.5} \mathrm{P}_{14.5}$ was prepared in a high-purity argon gas condition by melting high-purity Pd (99.5\%), Ni (99.9\%), Si (99.99\%) plates and $\mathrm{Ni}_{2} \mathrm{P}$ (99.95\%) powders (all purchased from Trillion Metals Co., Ltd.) as described in our previous work. ${ }^{26,28}$ Subsequently, the alloy in a quartz tube was purified with the fluxing $\mathrm{B}_{2} \mathrm{O}_{3}$ at $1373 \mathrm{~K}$ for $10 \mathrm{~h}$ to fully remove the impurities. Fully amorphous ingots were achieved by water quenching molten samples after fluxing. MG nanorod arrays were fabricated using a scalable top-down thermoplastic forming process. The flat MG plate was heated in the super-cooled liquid region above its glass transition temperature. Based on its thermal property (DSC curve), the PdNiSiP MG softened into a viscous liquid at the thermoplastic forming temperature of $350^{\circ} \mathrm{C}$ and flowed into the mold pattern under a constant load of $700 \mathrm{MPa}$. Commercially available nanoporous anodized aluminum oxide (AAO) molds (purchased from Shanghai Shangmu Technology Co., Ltd.) with $200 \mathrm{~nm}$ diameter pores were used as the template for nanoimprinting in this work. The MG nanorods were released from the AAO template by etching with $3 \mathrm{M} \mathrm{KOH}$ solution at $70{ }^{\circ} \mathrm{C}$. After about 30 minutes, AAO template was fully removed from the MG plate, followed by repeatedly rinsing with deionized water. The nanoimprinted PdNiSiP MG was cooled in the air till the temperature drops to room temperature, and the surface of MG nanorods immobilized in the AAO was significantly oxidized. The resulting oxidized MG NRAs electrode $(6 \mathrm{~mm} \times 6 \mathrm{~mm} \times 1$ $\mathrm{mm}$ ) was directly used in the electrochemical hydrogen production and in situ formed to hierarchical MG nanostructures by electroreduction in the early stage of electrolysis.

\section{Synthesis of OD-MG plate}

The oxidized MG plate, $6 \mathrm{~mm} \times 6 \mathrm{~mm} \times 1 \mathrm{~mm}$ in size, was formed by annealing flat $\mathrm{MG}$ plate at $280^{\circ} \mathrm{C}$ for 10 minutes. And the oxidized MG plate still keeps amorphous nature (Fig. S1 $\dagger$ ). The oxide-derived MG plate (OD-MG plate) was in situ formed by electrochemically reducing oxidized MG plate in initial HER electrolysis at the same conditions as the OD-MG NRAs electrode.

\section{Electrochemical measurement}

HER catalytic activity measurement was conducted in a standard three-electrode system controlled by a CHI660E electrochemistry workstation. All the experiments were performed in $0.5 \mathrm{M} \mathrm{H}_{2} \mathrm{SO}_{4}$ solution at room temperature. OD-MG NRAs was directly used as working electrode, a Pt plate (size $20 \times 15 \times 0.2$ $\mathrm{mm}$ ) as counter electrode and $\mathrm{Ag} / \mathrm{AgCl}$ ( $\mathrm{KCl}$ saturated) as reference electrode. The reference was calibrated against and converted to reversible hydrogen electrode (RHE) by the equation of $E(v s . \mathrm{RHE})=E(v s . \mathrm{Ag} / \mathrm{AgCl})+0.059 \times \mathrm{pH}+0.198 \mathrm{~V}$. For comparison, $\mathrm{Pt} / \mathrm{C}$ electrode was prepared by blending $10 \mathrm{mg} 20$ wt\% Pt/C and $30 \mu \mathrm{l}$ of $0.5 \mathrm{wt} \%$ Nafion solution with $250 \mu \mathrm{l}$ ethanol and $750 \mu \mathrm{l}$ deionized water in ultrasonication to form a homogenous dispersion. $10 \mu \mathrm{l}$ of the catalyst ink was then pipetted onto a $10 \mathrm{~mm} \times 10 \mathrm{~mm}$ glassy carbon paper. After obtaining a stable cycle between $-0.6 \mathrm{~V}$ and $0.1 \mathrm{~V}$, linear sweep voltammetry was carried out by sweeping the potential from $-0.3 \mathrm{~V}$ to $0 \mathrm{~V}\left(v s\right.$. RHE) at a scan rate of $5 \mathrm{mV} \mathrm{s}^{-1}$. Polarization curves and Tafel slopes of all the electrodes were derived from $90 \%$ iR compensation. Chronoamperometry was measured under a constant potential of $-0.3 \mathrm{~V}$. Cyclic voltammetry (CV) measurements were performed from -0.03 to $0.08 \mathrm{~V}$ at a scan rate of $10 \mathrm{mV} \mathrm{s}^{-1}$.

\section{Materials characterization}

The structures of all the samples were characterized by X-ray diffraction (XRD) using a Rigaku diffractometer with $\mathrm{Cu} \mathrm{K} \alpha$ radiation (40 kV and $200 \mathrm{~mA}$ ). The surface morphologies of ODMG plate and OD-MG NRAs electrode were inspected by ZEISS Merlin scanning electron microscope (SEM). The thermal property was studied by STA 449 F3 Jupiter at heating rate of 20 $\mathrm{K} \mathrm{min}^{-1}$. The surface composition and valence state of the ODMG plate and OD-MG NRAs were investigated by X-ray photoelectron spectroscopy (XPS), which is carried out using a spectrometer with $\mathrm{Al} \mathrm{K} \alpha$ radiation (ESCALAB 250Xi, Thermofisher Co.). All binding energies were calibrated with $\mathrm{C}$ 1s position $(284.8 \mathrm{eV})$ of contaminant carbon in the vacuum chamber of this instrument.

\section{Results and discussion}

Fig. 1 shows a schematic illustration of the fabrication of a novel $\mathrm{Pd}_{40.5} \mathrm{Ni}_{40.5} \mathrm{Si}_{4.5} \mathrm{P}_{14.5}$ nanostructured MG catalyst in five steps. (1) Assembling: firstly, the anodic aluminum oxide (AAO) template is placed on top of the flat $\mathrm{Pd}_{40.5} \mathrm{Ni}_{40.5} \mathrm{Si}_{4.5} \mathrm{P}_{14.5}$ MG plate. (2) Nanoimprinting: then MG plate is heated to its supercooled liquid region above its glass transition temperature, the MG fills the nanopores of the AAO template under the applied pressure and high yield of uniform nanorods is obtained in less than a minute of processing time. (3) Cooling: after that, we cooled the PdNiSiP MG nanorods immobilized in the nanopores of AAO in the air until the temperature drops down to room temperature. The thermal oxidation of palladium-based alloy containing nickel element is allowed in the air and becomes significant at temperatures above $300{ }^{\circ} \mathrm{C} .{ }^{30}$ Thus, the nickel at the surface of $\mathrm{Pd}_{40.5} \mathrm{Ni}_{40.5} \mathrm{Si}_{4.5} \mathrm{P}_{14.5}$ alloy nanorods was oxidized, which further confirmed by X-ray photoelectron spectroscopy measurement (Fig. 7). (4) Etching AAO: subsequently, the AAO template is dissolved in $\mathrm{KOH}$ solution to expose the oxidized MG nanorod arrays. Fig. 2a shows scanning electron microscope (SEM) image of oxidized MG nanorod arrays (oxidized MG NRAs) after nanoimprinting process. The SEM image shows that the MG nanorods of $200 \mathrm{~nm}$ diameter are well-isolated and highly-ordered and indicates lightly packed irregular NiO sheets that conformably cover the MG nanorod arrays. (5) Electroreduction: finally, the oxide-derived surface-modified MG nanorod arrays (OD-MG NRAs) was formed in situ via reduction 


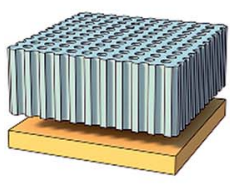

(1)

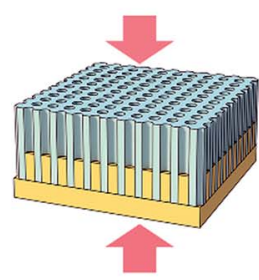

(2)

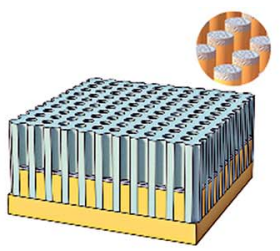

(3)

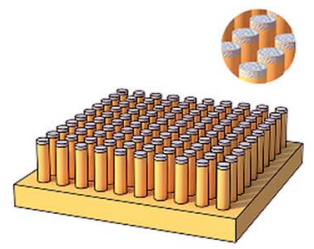

(4)

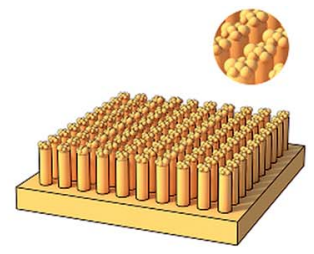

(5)

Fig. 1 Schematic illustration of the preparation process of the OD-MG NRAs. (1) Assembling; (2) nanoimprinting; (3) cooling; (4) etching AAO; (5) electroreduction.
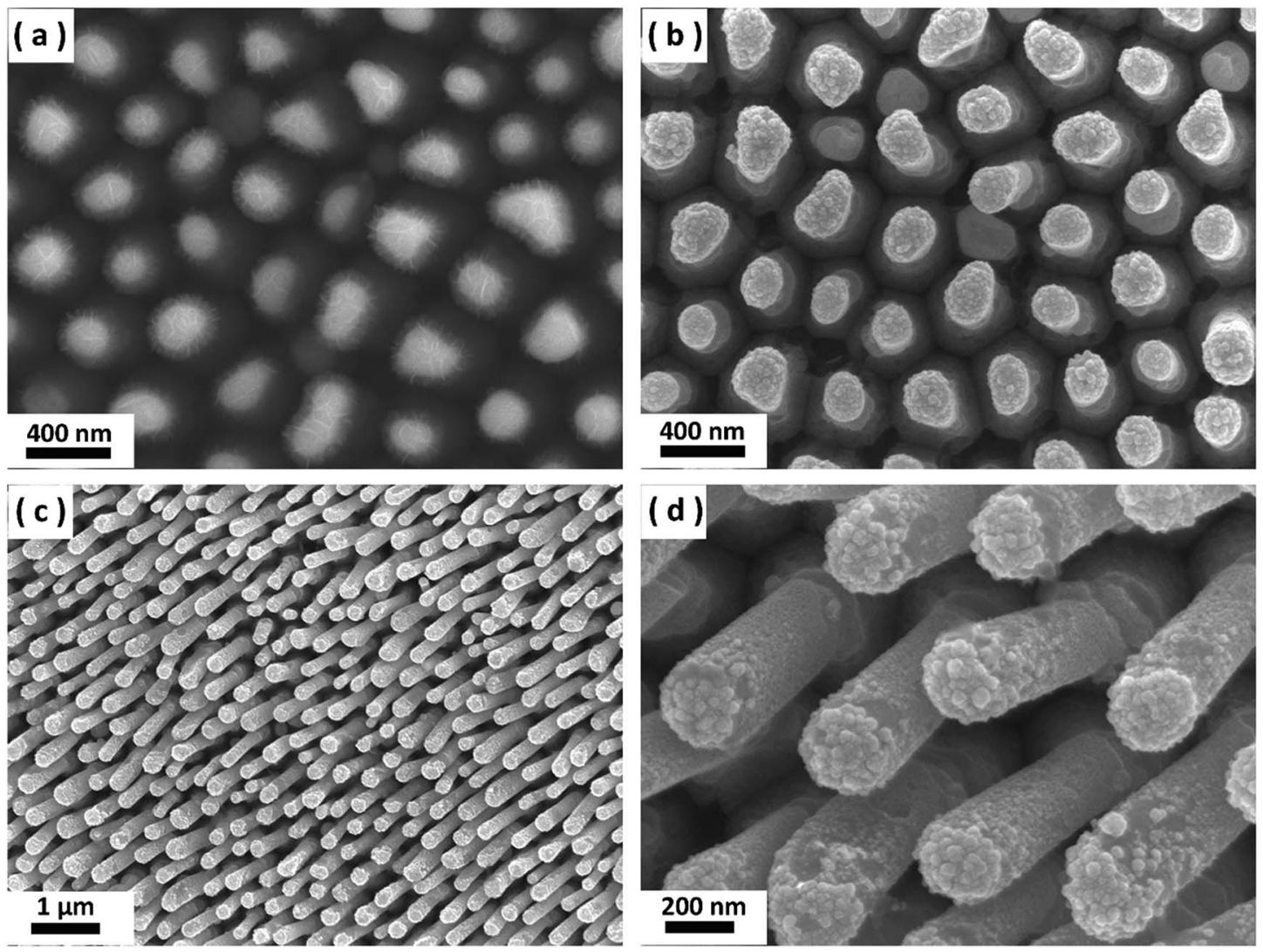

Fig. 2 SEM images of (a) oxidized MG NRAs after nanoimprinting, (b) OD-MG NRAs after electroreduction and (c and d) OD-MG NRAs electrode after $50000 \mathrm{~s}$ electrochemical testing taken at $45^{\circ}$

of the precursor NiO-coated MG nanorod arrays in the initial stage of hydrogen evolution electrocatalysis. OD-MG NRAs exhibited a high initial current density $\left(j_{\text {total }}\right)$ at $-0.1 \mathrm{~V} v s$. RHE (Fig. S2†), which stems from the reduction of the nickel oxide layer in the initial period of electrolysis. ${ }^{31-33}$ Subsequently, the current decreased to a point upon gradual consumption, because of a limited amount of the oxide. In contrast, the untreated flat MG showed constant low $j_{\text {total }}$. SEM image (Fig. 2b) indicated that after fully electroreduction of the nickel oxide film, the oxide-derived nanostructured MG electrode were comprised of agglomerated $\mathrm{Ni}$ nanoparticles on the heads of the MG nanorods. We also observed the abundant and dense metallic nanoparticles formed on the surface of oxide-derived
MG plate (OD-MG plate) after electroreduction in Fig. S3. $\dagger$ The X-ray photoelectron spectroscopy measurements (Fig. S4 $\dagger$ ) were performed to confirm the composition of OD-MG plate before and after electrolysis. The Ni 2p XPS spectrum (Fig. S4b†) revealed that the nickel oxide layer was transformed to metallic nanoparticles without any $\mathrm{Ni}$ oxide. By the simple synthesis technique, the surface-modified MG nanorod array electrode resulting from metal oxide possess higher specific surface area compared to that of untreated MG, which play a major role in the enhancement of catalytic activity.

Fig. 3 shows the XRD patterns of MG plate, oxidized MG NRAs and OD-MG NRAs, which all displayed a broad diffraction maximum with the absence of the characteristic crystalline 


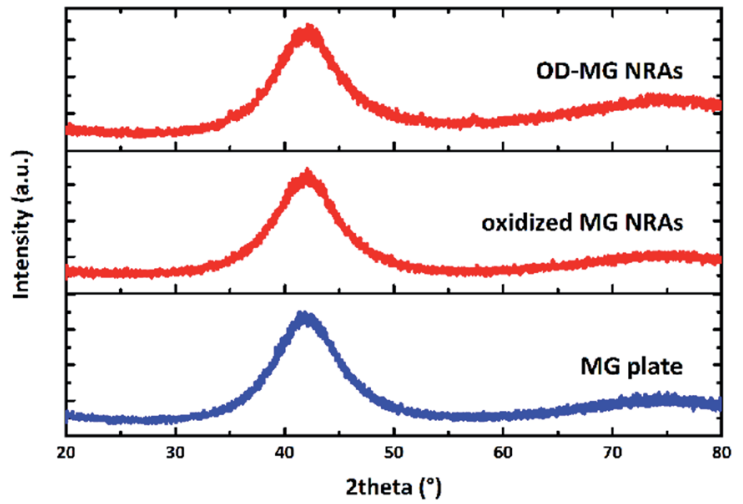

Fig. 3 XRD patterns of MG plate, oxidized MG NRAs and OD-MG NRAs (from the bottom up).

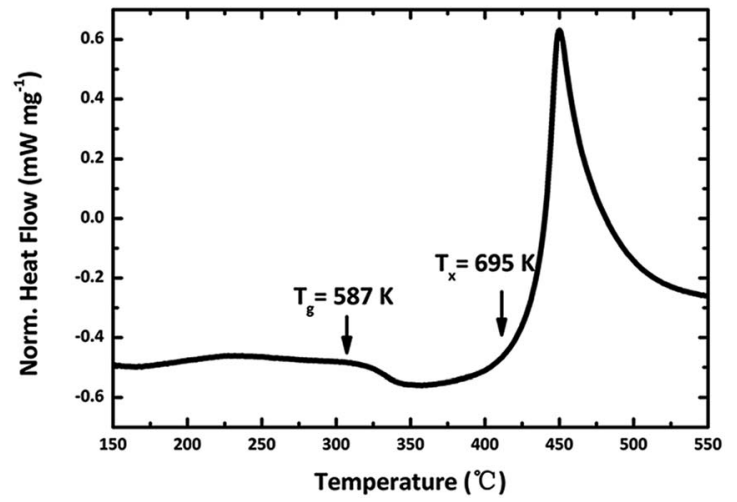

Fig. 4 DSC curve of the $\mathrm{Pd}_{40.5} \mathrm{Ni}_{40.5} \mathrm{Si}_{4.5} \mathrm{P}_{14.5}$ MG plate.

diffraction peaks, confirming the amorphous nature of the nanostructured MG electrode during the whole process.

The thermal property of the PdNiSiP MG was analyzed by differential scanning calorimetry (DSC). As shown in Fig. 4, the MG exhibits typical glassy features with increasing temperature, such as endothermic reaction of glass transition, wide supercooled liquid region and sharp exothermic peak of crystallization. From the DSC curve, the glass transition temperature $T_{\mathrm{g}}$ and crystallization temperature $T_{\mathrm{x}}$ were determined to be $\sim 587$ $\mathrm{K}$ and $695 \mathrm{~K}$, respectively.

We evaluated the electrochemical characteristics of the untreated flat MG plate (MG plate), oxide-derived surfacemodified MG nanorod arrays (OD-MG NRAs) and commercial $\mathrm{Pt} / \mathrm{C}$ electrodes towards the HER catalytic performance using a standard three-electrode configuration in $0.5 \mathrm{M} \mathrm{H}_{2} \mathrm{SO}_{4}$. In the initial period of electrolysis, OD-MG NRAs electrode was directly formed in situ by the corrosion of the nickel oxide formed on the MG nanorod. Then linear sweep voltammetry were performed in the electrochemical cell. Polarization curves ( $i R$-corrected) of the geometric current density (j) plotted against potential show the HER activity of the MG plate and OD-MG NRAs along with Pt/C (Fig. 5a and b). Fig. 5a shows that the untreated flat MG plate, OD-MG NRAs and Pt/C electrodes achieved geometric current densities of $-10 \mathrm{~mA} \mathrm{~cm} \mathrm{~cm}^{-2}$ at low overpotentials of
$138 \mathrm{mV}, 63 \mathrm{mV}$ and $32 \mathrm{mV}$, respectively, versus the reversible hydrogen electrode (RHE). Despite a nominal change in onset potential, a $54 \%$ decline of overpotential at $10 \mathrm{~mA} \mathrm{~cm}{ }^{-2}$ was measured when the flat MG electrode was modified into nanorod array structure. The comparison in Fig. 5b highlights that oxide-reduced surface-modified MG NRAs morphology clearly plays a key role in catalytic performance. The untreated flat MG plate requires an applied overpotential of $227 \mathrm{mV} v s$. RHE for $j=50 \mathrm{~mA} \mathrm{~cm}{ }^{-2}$, while the OD-MG NRAs require only $99 \mathrm{mV} v$ s. RHE. This enhancement of electrochemical hydrogen production was partially attributed to the higher surface area of OD-MG NRAs. We have determined the electrochemically active surface area (ECSA) of the flat MG plate and OD-MG NRAs electrodes from estimating the amount of absorbed hydrogen in the hydrogen underpotential deposition (H-UPD) region of the cyclic voltammogram. The detailed calculation method of ECSA is provided in ESI. $\dagger$ As shown in Fig. 5c, there are two H-UPD peaks on the surfaces of the two electrodes in the lowpotential region. Careful inspection reveals that the two $\mathrm{H}$ UPD peak-potentials of the OD-MG NRAs electrode $(-6 \mathrm{mV}$ and $39 \mathrm{mV})$ are higher than those of the flat MG plate $(-14 \mathrm{mV}$ and $32 \mathrm{mV}$ ). The higher potential of the H-UPD peaks indicates that hydrogen is easily adsorbed on the OD-MG NRAs surface. The ECSA is calculated from the area of the electrochemical peaks associated with the stripping of the absorbed hydrogen from the electrode surface and the resulting ECSA for MG plate and OD-MG NRAs are $20.3 \mathrm{~cm}^{2}$ and $47.4 \mathrm{~cm}^{2}$, respectively. This comparison reveals that the oxide-derived surface-modified MG NRAs more than doubles the effective surface area compared to the untreated flat MG plate. After normalization to the ECSA, the specific activity of OD-MG NRAs is also higher than that of untreated flat MG plate (Fig. S6 $†$ ), which is possibly stemming from a combination of high intrinsic activity correlated with the oxide-derived surface as well as the nanorods architecture leading to improved access to active sites. From the extrapolation of the linear region of a plot of overpotential versus $\log (-j)$ (Fig. 5d), we obtained Tafel slopes of 73.6, 42.6 and $29.2 \mathrm{mV}$ $\mathrm{dec}^{-1}$ for MG plate, OD-MG NRAs and Pt/C electrodes, respectively. The OD-MG NRAs electrode exhibited a smaller Tafel slope of $42.6 \mathrm{mV} \mathrm{dec}{ }^{-1}$, suggesting a two-electron transfer process on the oxide-derived nanorod surface following a Volmer-Heyrovsky mechanism, where electrochemical desorption of hydrogen is regarded as the rate-limiting step. ${ }^{34}$ This value is much lower than that of untreated flat MG plate, indicating a faster electron transfer to a $\mathrm{H}_{2}$ molecule in a nanostructured MG catalyst. These results all demonstrated the excellent HER electrochemical activity and kinetic property of the OD-MG NRAs cathode.

Long-term stability is an important parameter for a viable HER catalyst. To investigate stability under electrocatalytic operation, we have measured the durability of OD-MG NRAs by monitoring the current density during continuous electrolysis at -0.3 V. $20 \mathrm{wt} \% \mathrm{Pt} / \mathrm{C}$ was also tested for comparison. The normalized current density versus time data provided in the Fig. 6 shows that the current density of OD-MG NRAs decreases at the early stage of testing and reaches about $85 \%$ of the initial value around $5000 \mathrm{~s}$. Surprisingly, the efficiency of OD-MG NRAs 

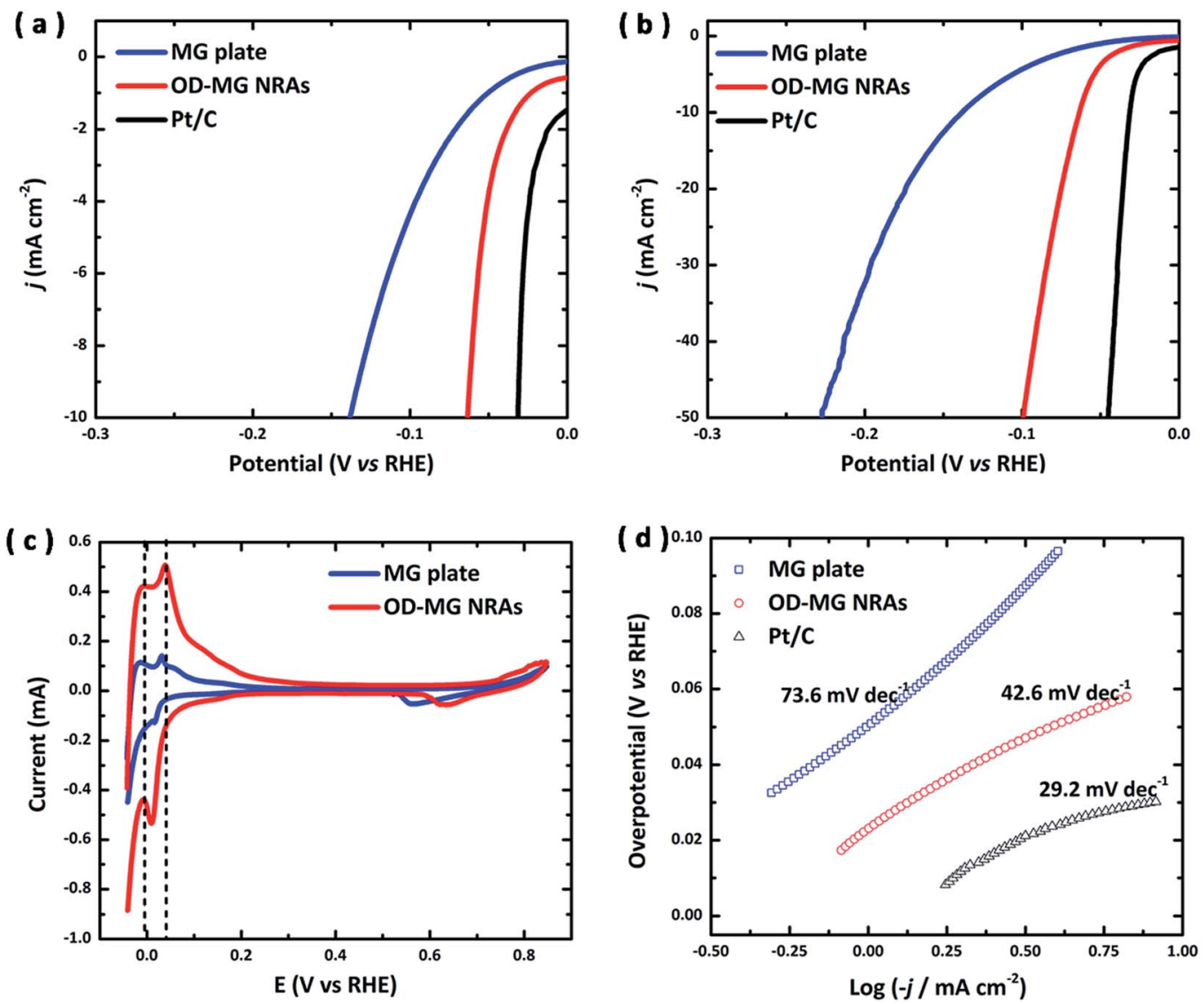

Fig. 5 Electrochemical characterization of MG plate, OD-MG NRAs and Pt/C electrodes for HER electrocatalysis. Polarization curves at (a) lower and (b) higher applied overpotentials. (c) Cyclic voltammograms of MG plate and OD-MG NRAs. (d) Tafel analysis of the data presented in panel a.

is thriving and the current density increases to $100 \%$ after $40000 \mathrm{~s}$ test. Even after long-term electrochemical testing, the OD-MG NRAs catalyst stayed no change in the morphology (Fig. 2c and d) and kept the amorphous nature (Fig. S7 $\dagger$ ).

To better understand the impressive stability of the OD-MG NRAs catalyst, X-ray photoelectron spectroscopy (XPS) measurements were performed to investigate the composition and valence state on the surface of the samples during the electrolysis test. Fig. 7a presents the spectra of Pd 3d, which indicates that metallic Pd predominates in the fresh sample

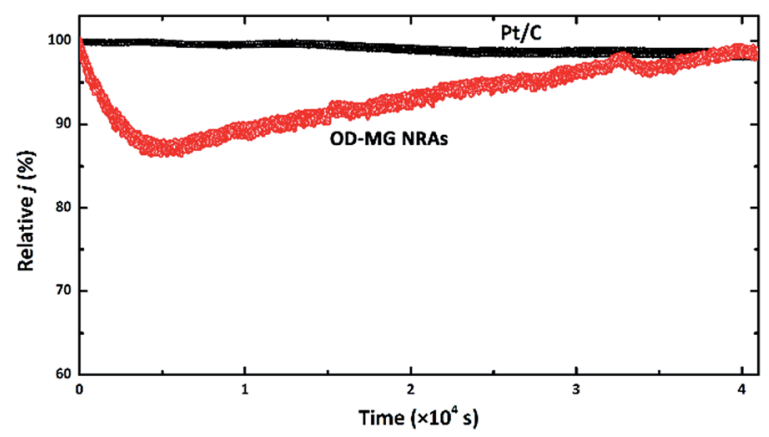

Fig. 6 Variations of the percentages of initial current density at a static potential of $-0.3 \mathrm{~V}$ versus time. with traces of PdO, while only Pd remains on the surface after $50000 \mathrm{~s}$. As noted in Fig. 7b, the peaks in Ni 2p spectrum (see below) can be assigned to oxidized state $\mathrm{Ni}^{2+}$ on the surface, which proves that the surface of the nanoimprinted PdNiSiP alloy have been oxidized due to the exposure to air during cooling process resulting in a layer of $\mathrm{NiO}$ at the surface. In the region detected by XPS, the normalized atomic percentage of Pd and $\mathrm{Ni}$ are $4.3 \%$ and $95.7 \%$, respectively, indicating a significant segregation of nickel onto the surface under the cooling process of nanoimprinted MG. Due to the mild oxidation of MG nanorod and thus thin layer of $\mathrm{NiO}$, we proposed that the $\mathrm{NiO} / \mathrm{Ni}$ interfaces and small amount of metallic Pd might be synergistically active sites for hydrogen evolution in the initial period of HER. ${ }^{5}$ Along with electrolysis, these oxidized states disappeared and thin NiO nanosheets reducted to metallic Ni nanoparticles. In the Ni 2p spectrum (see above), metallic Ni had two typical peaks: $853.8 \mathrm{eV} 2 \mathrm{p}_{3 / 2}$ and $871.1 \mathrm{eV} 2 \mathrm{p}_{1 / 2}{ }^{30}$ After long-term electrochemical operation, the surface composition has changed; the normalized atomic percentage of Pd and $\mathrm{Ni}$ are $12.2 \%$ and $87.8 \%$, respectively. It is distinct that selective dealloying happens during the testing process, due to the electronegativity difference. Therefore, the surface of OD-MG NRAs is enriched with catalytically active palladium. The reduction of $\mathrm{NiO}$ and dealloying of $\mathrm{Ni}$ together induced a novel hierarchical nanostructures with a Pd-abundant surface and 

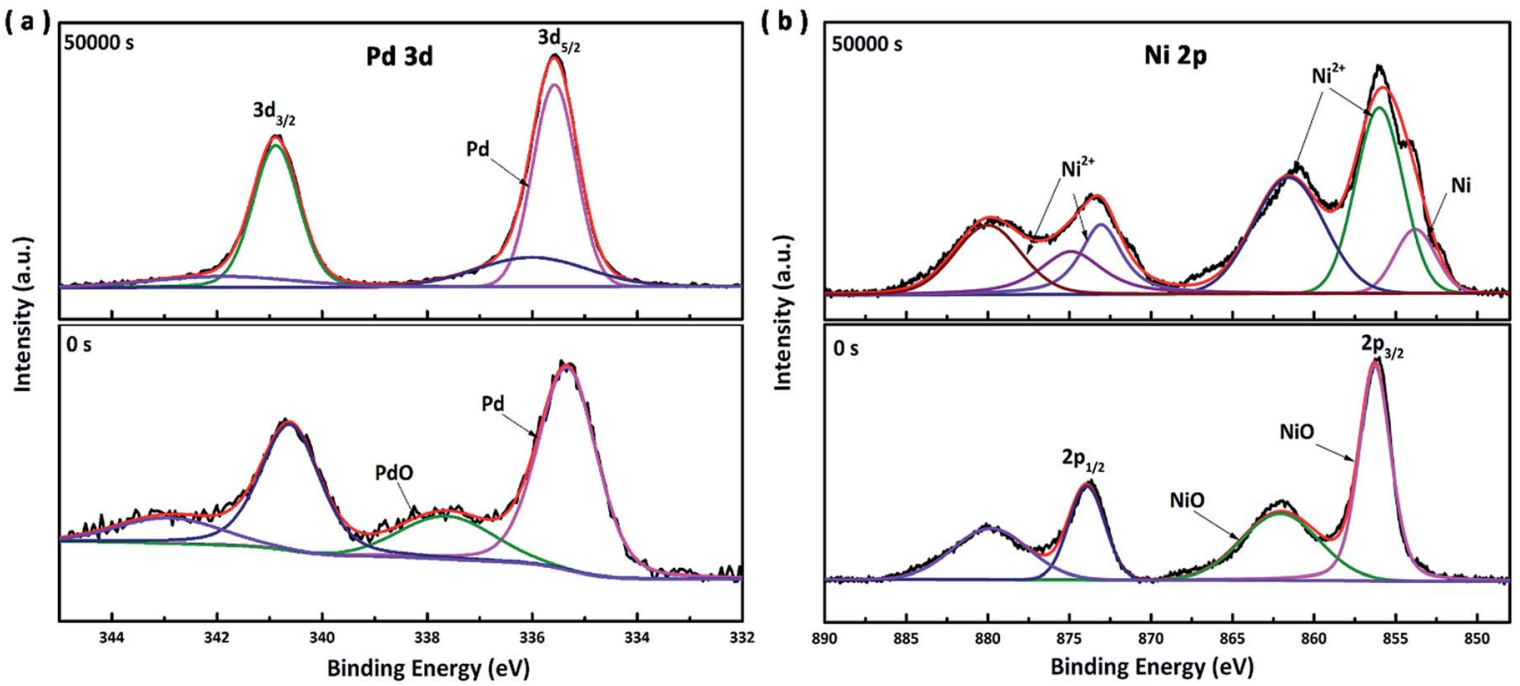

Fig. 7 (a) Pd 3d spectra and (b) Ni 2p spectra of the OD-MG NRAs before (see below) and after 50000 s test (see above).

large surface area, which is crucial to understanding the impressive HER catalysis of the MG nanodome..$^{35,36}$

\section{Conclusions}

In summary, we demonstrate OD-MG NRAs as a novel highperformance catalyst for robust electrochemical hydrogen production. Our nanostructure synthesis method is easy, scalable and economical by combining thermoplastic forming with electrochemical reduction. In addition, our oxide-derived surface-modification method is a unique strategy to create and optimize electrochemically active surfaces. The oxidederived surface-modified $\mathrm{Pd}_{40.5} \mathrm{Ni}_{40.5} \mathrm{Si}_{4.5} \mathrm{P}_{14.5}$ MG NRAs electrode achieved current density of $10 \mathrm{~mA} \mathrm{~cm} \mathrm{~cm}^{-2}$ at overpotentials as low as $63 \mathrm{mV}$ with unique self-stabilizing stability, which is among the most active HER catalysts reported so far. We expected that oxide-derived nanostructured metallic-glass and techniques established here can be further extended for a number of enticing applications.

\section{Acknowledgements}

This work is supported by the National Natural Science Foundation of China (Grant No. 51571127).

\section{References}

1 G. W. Crabtree, M. S. Dresseihaus and M. V. Buchanan, Phys. Today, 2004, 57, 39-44.

2 M. S. Dresselhaus and I. L. Thomas, Nature, 2001, 414, 332337.

3 J. R. Mckone, S. C. Marinescu, B. S. Brunschwig, J. R. Winkler and H. B. Gray, Chem. Sci., 2014, 5, 865-878.

4 C. G. Morales-guio, L. Stern and X. Hu, Chem. Soc. Rev., 2014, 43, 6555-6569.
5 M. Gong, W. Zhou, M.-C. Tsai, J. Zhou, M. Guan, M.-C. Lin, B. Zhang, Y. Hu, D.-Y. Wang, J. Yang, S. J. Pennycook, B.-J. Hwang and H. Dai, Nat. Commun., 2014, 5, 4695.

6 R. Liu, S. Gu, H. Du and C. M. Li, J. Mater. Chem. A, 2014, 2, 17263-17267.

7 Z. Shi, Y. Wang, H. Lin, H. Zhang, M. Shen, S. Xie, Y. Zhang, Q. Gao and Y. Tang, J. Mater. Chem. A, 2016, 4, 6006-6013.

8 Y. Huang, Y.-E. Miao, J. Fu, S. Mo, C. Wei and T. Liu, J. Mater. Chem. A, 2015, 3, 16263-16271.

9 X. Cao, Y. Han, C. Gao, Y. Xu, X. Huang, M. Willander and N. Wang, Nano Energy, 2014, 9, 301-308.

10 Q. Lu, G. S. Hutchings, W. Yu, Y. Zhou, R. V. Forest, R. Tao, J. Rosen, B. T. Yonemoto, Z. Cao, H. Zheng, Q. Xiao, F. Jiao and J. G. Chen, Nat. Commun., 2015, 6, 6567.

11 Z. W. Seh, J. Kibsgaard, C. F. Dickens, I. Chorkendorff, J. K. Nørskov and T. F. Jaramillo, Science, 2017, 355, 146-158.

12 J. D. Benck, T. R. Hellstern, J. Kibsgaard, P. Chakthranont and T. F. Jaramillo, ACS Catal., 2014, 4, 3957-3971.

13 B. Rezaei, M. Mokhtarianpour and A. A. Ensafi, Int. J. Hydrogen Energy, 2015, 40, 6754-6762.

14 L. Genovese, C. Cocchiara, S. Piazza, C. Sunseri and R. Inguanta, Mater. Res. Bull., 2017, 86, 10-18.

15 Y. C. Hu, Y. Z. Wang, R. Su, C. R. Cao, F. Li, C. W. Sun, Y. Yang, P. F. Guan, D. W. Ding, Z. L. Wang and W. H. Wang, Adv. Mater., 2016, 28, 10293-10297.

16 M. Zhao, K. Abe, S. Yamaura, Y. Yamamoto and N. Asao, Chem. Mater., 2014, 26, 1056-1061.

17 Z. Shan, Y. Liu, Z. Chen, G. Warrender and J. Tian, Int. J. Hydrogen Energy, 2008, 33, 28-33.

18 M. Carmo, R. C. Sekol, S. Ding, G. Kumar, J. Schroers and A. D. Taylor, ACS Nano, 2011, 5, 2979-2983.

19 B. W. An, E.-J. Gwak, K. Kim, Y.-C. Kim, J. Jang, J.-Y. Kim and J.-U. Park, Nano Lett., 2016, 16, 471-478.

20 R. C. Sekol, G. Kumar, M. Carmo, F. Gittleson, N. Hardestydyck, S. Mukherjee, J. Schroers and A. D. Taylor, Small, 2013, 9, 2081-2085. 
21 A. L. Greer, Science, 1995, 267, 1947-1953.

22 A. Inoue, Acta Mater., 2000, 48, 279-306.

23 W. L. Johnson, MRS Bull., 1999, 1, 42-56.

24 J. Schroers, Adv. Mater., 2010, 22, 1566-1597.

25 G. Kumar, H. X. Tang and J. Schroers, Nature, 2009, 457, 868872.

26 X. Liu, Y. Shao, J. Li, N. Chen and K.-F. Yao, J. Alloys Compd., 2014, 605, 7-11.

27 M. Hasan, J. Schroers and G. Kumar, Nano Lett., 2015, 15, 963-968.

28 X. Liu, Y. Shao, Y. Tang and K.-F. Yao, Sci. Rep., 2014, 4, 1-7.

29 G. Doubek, R. C. Sekol, J. Li, W.-H. Ryu, F. S. Gittleson, S. Nejati, E. Moy, C. Reid, M. Carmo, M. Linardi, P. Bordeenithikasem, E. Kinser, Y. Liu, X. Tong, C. O. Osuji, J. Schroers, S. Mukherjee and A. D. Taylor, Adv. Mater., 2016, 28, 1940-1949.

30 Z. Song, X. Bao, U. Wild, M. Muhler and G. Ertl, Appl. Surf. Sci., 1998, 134, 31-38.
31 M. Ma, B. J. Trzesniewski, J. Xie and W. A. Smith, Angew. Chem., Int. Ed., 2016, 55, 1-6.

32 C. W. Li and M. W. Kanan, J. Am. Chem. Soc., 2012, 134, 7231-7234.

33 Y. Chen, C. W. Li and M. W. Kanan, J. Am. Chem. Soc., 2012, 134, 19969-19972.

34 J. K. Norskov, T. Bligaard, A. Logadottir, J. R. Kitchin, J. G. Chen, S. Pandelov and U. Stimming, J. Electrochem. Soc., 2005, 152, 23-26.

35 Y. Zeng, S. Yang, H. Xiang, X. Dong, L. Chen, M. W. Chen, A. Inoue, X. Zhang and J. Jiang, Intermetallics, 2015, 61, 66-71.

36 M. Li, Z. Zhao, T. Cheng, A. Fortunelli, C.-Y. Chen, R. Yu, Q. Zhang, L. Gu, B. V Merinov, Z. Lin, E. Zhu, T. Yu, Q. Jia, J. Guo, L. Zhang, W. A. Goddard, Y. Huang and X. Duan, Science, 2016, 354, 1414-1419. 